\title{
Mechanisms of elastic-plastic deformation in magnesium alloy studied using neutron diffraction and crystallite group method
}

\author{
P. Kot ${ }^{1}$, A. Baczmański ${ }^{1}$, M. Wroński ${ }^{1}$, S. Wroński ${ }^{1}$, Ch. Scheffzük ${ }^{2}$, G. Bokuchava ${ }^{2}$, V. Sikolenko ${ }^{2}$ \\ ${ }^{1} A G H$ University of Science and Technology, WFiIS, al. Mickiewicza 30, 30-052 Kraków, Poland \\ ${ }^{2}$ Joint Institute for Nuclear Research, Frank Laboratory of Neutron Physics, Joliot-Curie 6, Dubna 141980, Russia, \\ przemyslaw.kot@fis.agh.edu.pl
}

Important problem studied in this work is the anisotropy of mechanical properties for textured polycrystalline materials [1]. The mechanical behaviour during in-situ loading tests for magnesium AZ-31 alloy was studied using neutron diffraction. The lattice strains were measured during tensile by using angle-dispersive neutron diffraction (TKSN 400 at NPI in Řež, Czech Republic) and changing sample orientation with respect to the scattering vector [2]. The measurements were done for sets of poles (Fig. 1a) corresponding to different orientations of the grains in strongly textured $\mathrm{Mg}$ alloy. Subsequent experiment was performed using time of flight (TOF) neutron diffraction at the pulsed reactor IBR-2 in Joint Institute for Nuclear Research (Dubna, Russia), using EPSILON-MDS instrument equipped with 9 detectors. The experiments allowed to develop an experimental methodology based on the so-called crystallite group method [3] in order to determine the evolution of the stresses localised in polycrystalline grains having different crystallographic orientations. The components of stress tensor were determined directly from measured lattice strains corresponding to chosen orientations of crystallite lattice.

It was found that the crystallites having two main orientations, named A and B, are harder when compared with other ones (Fig. 1a,b). For these orientations the basal slip system cannot be activated because the load is applied in direction parallel to the basal plane. Orientation B was completely transformed to twins (having T orientation, cf. Fig. 1b) during the compression test. In the case of the soft orientations $\mathrm{C}$ and $\mathrm{D}$, the direction of the load is inclined from the basal plane, i.e. the basal system can be activated. Using the experimental data the evolution of stress tensor and von Mises stress were determined for selected groups of grains. A large difference in the hardness of crystallites having different lattice orientations (Fig. 1c) was found. The highest von Mises stress appeared on twins, which was compensated by low stresses localised on soft orientations $\mathrm{C}$ and $\mathrm{D}$.

The novelty of our study is in original methodology used for direct determining of stress tensor for groups of polycrystalline grains having different orientations (especially for preferred texture orientations). The stress evolution measured during sample loading allowed us to find out the critical resolved shear stress (CRSS) values for different slip systems and twinning process.

a)
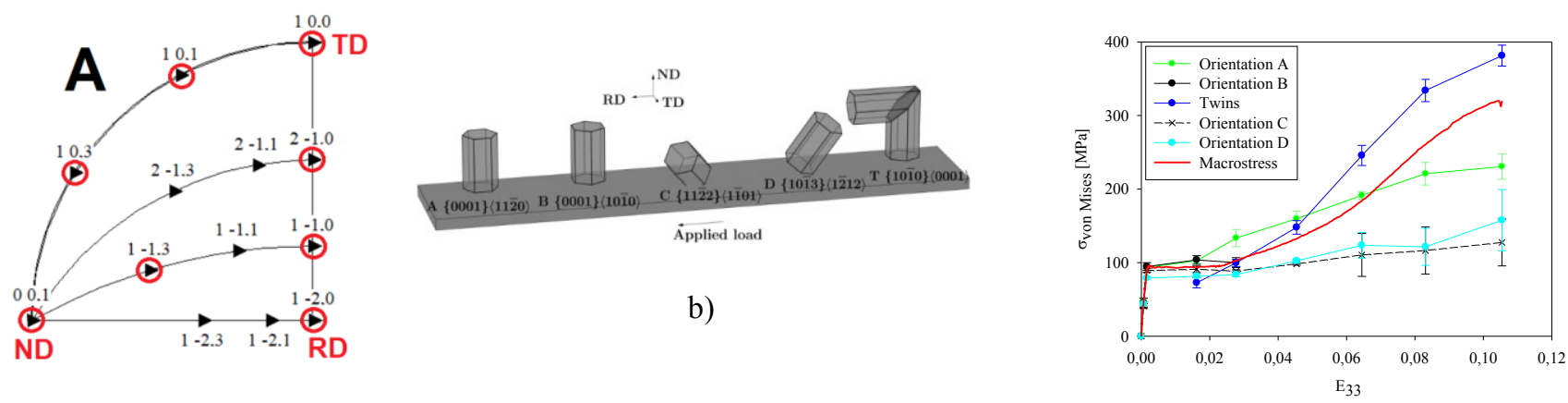

Figure 1. a) The groups of poles for which lattice strains were measured (marked in red) for given crystallite orientation A. b) Selected orientations of crystallites A B, C, D and T - twin. c) Evolution of von Mises stress for selected orientations. The red line corresponds to the macroscopic stress-strain curve.

[1] Wang, H., Clausen, B., Capolungo, L., Beyerlein, I.J., Wang, J., Tomé, C.N., Int. J. Plast. 79 (2016) $275-292$.

[2] Baczmański, A., Kot, B., Wroński, S., Wróbel, M. Wroński, M., Pilch, J., Muzyka, M., et al., Mater. Sci. Eng. A. 801 (2021) 140355

[3] Hauk, V., Structural and Residual Stress Analysis by Nondestructive Methods, 1997

\section{Keywords: Neutron diffraction; Stress localisation; Slip systems; Twinning; Elastoplastic deformation; Magnesium alloy}

This work was partially supported by grants from the National Science Centre, Poland (NCN) No. UMO-2017/25/B/ST8/00134 Measurements were partly carried out at the CANAM infrastructure of the NPI CAS Rez with the use of infrastructure Reactors LVR15 and LR-0, which is financially supported by the Ministry of Education, Youth and Sports - project LM2018120.

The TOF neutron diffraction experiments performed using EPSILON instrument were co-financed by the Plenipotentiary Representative of the Government of the Republic of Poland at JINR in Dubna under the Project No. PWB/168-22/2021.

P. Kot has been partly supported by the EU Project POWR.03.02.00-00-1004/16.

Acta Cryst. (2021), A77, C220 